International Journal of Applied Mathematics

Volume 30 No. $6 \quad 2017,501-514$

ISSN: 1311-1728 (printed version); ISSN: 1314-8060 (on-line version)

doi: http://dx.doi.org/10.12732/ijam.v30i6.4

\title{
A NOVEL SUBCLASS OF UNIVALENT FUNCTIONS INVOLVING OPERATORS OF FRACTIONAL CALCULUS
}

\author{
P.N. Kamble ${ }^{1}$, M.G. Shrigan ${ }^{2}$, H.M. Srivastava ${ }^{3} \S$ \\ ${ }^{1}$ Department of Mathematics \\ Dr. Babasaheb Ambedkar Marathwada University \\ Aurangabad, 431004, Maharashtra State, INDIA \\ ${ }^{2}$ Department of Mathematics \\ Dr. D.Y. Patil School of Engineering and Technology \\ Pune 412205, Maharashtra State, INDIA \\ ${ }^{3}$ Department of Mathematics and Statistics \\ University of Victoria \\ Victoria, British Columbia V8W 3R4, CANADA \\ ${ }^{3}$ Department of Medical Research \\ China Medical University Hospital
}

China Medical University, Taichung 40402, Taiwan, REPUBLIC OF CHINA

\begin{abstract}
In this paper, we introduce and investigate a novel class of analytic and univalent functions with negative Taylor-Maclaurin coefficients in the open unit disk. For this function class, we obtain characterization and distortion theorems as well as the radii of close-to-convexity, starlikeness and convexity by using techniques involving operators of fractional calculus.
\end{abstract}

AMS Subject Classification: 30C45, 30C50, 26A33

Key Words: analytic functions, fractional derivative operator, starlike functions, close-to-convex functions, convex functions

\section{Introduction}

Let $\mathcal{T}_{n}$ denote the class of functions $f(z)$ of the form:

$\begin{array}{ll}\text { Received: October 21, } 2017 & \text { (c) } 2017 \text { Academic Publications }\end{array}$

${ }^{\S}$ Correspondence author 


$$
f(z)=z-\sum_{k=n+1}^{\infty} a_{k} z^{k} \quad\left(a_{k} \geqq 0 ; n \in \mathbb{N}=\{1,2,3, \cdots\}\right),
$$

which are analytic and univalent in the open unit disk given by

$$
\mathbb{U}=\{z: z \in \mathbb{C} \text { and }|z|<1\}
$$

We denote by $\mathcal{T}_{n}(\lambda, \mu, \eta)$ the subclass of functions $f(z)$ in $\mathcal{T}_{n}$ which also satisfy the following inequality:

$$
\begin{gathered}
\left|\frac{z J_{0, z}^{\lambda+1, \mu+1, \eta+1} f(z)-(1-\mu) J_{0, z}^{\lambda, \mu, \eta} f(z)}{\lambda z J_{0, z}^{\lambda+1, \mu+1, \eta+1} f(z)+(1-\lambda) J_{0, z}^{\lambda, \mu, \eta} f(z)}\right|<\alpha \\
(z \in \mathbb{U} ; 0 \leqq \mu<1 ; 0 \leqq \lambda \leqq 1 ; 0<\alpha \leqq 1 ; \mu, \eta \in \mathbb{R}),
\end{gathered}
$$

where $J_{0, z}^{\lambda, \mu, \eta}$ denotes an operator of fractional derivative given by Definition 5 below.

The purpose of the present paper is to study the above-defined (presumably new) subclass $\mathcal{T}_{n}(\lambda, \mu, \eta)$ of analytic and univalent functions with negative Taylor-Maclaurin coefficients involving a certain fractional calculus operator. In Section 1, we introduce the necessary details of this subclass of analytic and univalent functions. Section 2 gives details about the fractional derivative and integral operators which are involved in our investigation. In Section 3, several preliminary results related to fractional derivative operators have been discussed. In Section 4, we investigate the characterization theorem for the functions belonging to the subclass $\mathcal{T}_{n}(\lambda, \mu, \eta)$. Section 5 gives a distortion theorem for the subclass $\mathcal{T}_{n}(\lambda, \mu, \eta)$. Section 6 gives the radii of close-to-convexity, starlikeness and convexity by using operators of fractional calculus.

\section{Operators of Fractional Calculus}

Fractional calculus is one of the most intensively developing areas of the mathematical analysis. The fractional calculus operators have gone deep across into the realm of the theory of univalent functions. Various operators of fractional calculus have been studied in the literature rather extensively. We find it to be convenient to recall here the following definitions (cf., e.g., [4], [5] and [10]). 
Definition 1. (Fractional Integral Operator) The fractional integral of order $\lambda$ is defined, for a function $f(z)$, by

$$
D_{z}^{-\lambda} f(z)=\frac{1}{\Gamma(\lambda)} \int_{0}^{z}(z-\zeta)^{\lambda-1} f(\zeta) d \zeta \quad(\lambda>0),
$$

where $\lambda>0, f(z)$ is an analytic function in a simple-connected region of the complex $z$-plane containing the origin and the multiplicity of $(z-\zeta)^{\lambda-1}$ is removed by requiring $\log (z-\zeta)$ to be real when $z-\zeta>0$.

Definition 2. (Fractional Derivative Operator) The fractional derivative of order $\lambda$ is defined, for a function $f(z)$, by

$$
D_{z}^{\lambda} f(z)=\frac{1}{\Gamma(1-\lambda)} \frac{d}{d z} \int_{0}^{z}(z-\zeta)^{-\lambda} f(\zeta) d \zeta \quad(0 \leqq \lambda<1),
$$

where $f(z)$ is constrained, and the multiplicity of $(z-\zeta)^{-\lambda}$ is removed, as in Definition 1.

Definition 3. (Extended Fractional Derivative Operator) Under the hypotheses of Definition 2, the fractional derivative of order $n+\lambda$ is defined, for a function $f(z)$, by

$$
D_{z}^{n+\lambda} f(z)=\frac{d^{n}}{d z^{n}} D_{z}^{\lambda} f(z) \quad\left(0 \leqq \lambda<1 ; n \in \mathbb{N}_{0}:=\mathbb{N} \cup\{0\}\right) .
$$

Let ${ }_{2} F_{1}(a, b ; c ; z)$ be the Gauss hypergeometric function defined for $z \in \mathbb{U}$ by (see, for example, $[9$, p. 18])

$$
{ }_{2} F_{1}(a, b ; c ; z)=\sum_{k=0}^{\infty} \frac{(a)_{k}(b)_{k}}{(c)_{k}} \frac{z^{k}}{k !} \quad(z \in \mathbb{U}),
$$

where $(\lambda)_{k}$ denotes the Pochhammer symbol defined by

$$
(\lambda)_{k}=\frac{\Gamma(\lambda+k)}{\Gamma(\lambda)}=\left\{\begin{array}{ll}
1 & (k=1) \\
\lambda(\lambda+1) \cdots(\lambda+k-1) & (\forall k \in \mathbb{N})
\end{array} .\right.
$$

Making use of the Gauss hypergeometric function ${ }_{2} F_{1}(a, b ; c ; z)$ given by (7), Srivastava et al. [12] introduced the fractional integral operator $I_{0, z}^{\lambda, \mu, \eta}$ defined below. 
Definition 4. Let $\lambda \in \mathbb{R}_{+}=(0, \infty)$ and $\mu, \eta \in \mathbb{R}$. Then, in terms of the familiar Gauss's hypergeometric function ${ }_{2} F_{1}(a, b ; c ; z)$ given by $(7)$, the fractional integral operator $I_{0, z}^{\lambda, \mu, \eta}$ is defined by

$$
I_{0, z}^{\lambda, \mu, \eta} f(z)=\frac{z^{-\lambda-\mu}}{\Gamma(\lambda)} \int_{0}^{z}(z-\zeta)^{\lambda-1} f(\zeta){ }_{2} F_{1}\left(\lambda+\mu,-\eta ; \lambda ; 1-\frac{\zeta}{z}\right) d \zeta
$$

where the function $f(z)$ is analytic in a simply-connected region of the complex $z$-plane containing the origin, with the order given by

$$
f(z)=O\left(|z|^{\varepsilon}\right) \quad(z \rightarrow 0)
$$

for

$$
\varepsilon>\max \{0, \mu-\eta\}-1),
$$

and the multiplicity of $(z-\zeta)^{\lambda-1}$ is removed by requiring $\log (z-\zeta)$ to be real when $z-\zeta>0$.

Definition 5. The fractional derivative operator $J_{0, z}^{\lambda, \mu, \eta}$ is defined by

$$
\begin{aligned}
J_{0, z}^{\lambda, \mu, \eta} f(z)=\frac{d}{d z} & \left(\frac{z^{\lambda-\mu}}{\Gamma(1-\lambda)} \int_{0}^{z}(z-\zeta)^{-\lambda} f(\zeta)\right. \\
& \left.\cdot{ }_{2} F_{1}\left(\mu-\lambda, 1-\eta ; 1-\lambda ; 1-\frac{\zeta}{z}\right) d \zeta\right) \\
& (0 \leqq \lambda<1 ; \mu, \eta \in \mathbb{R}),
\end{aligned}
$$

where the function $f(z)$ is analytic in a simply-connected region of the complex $z$-plane containing the origin, with the same order as that given by (10), and multiplicity of $(z-\zeta)^{-\lambda}$ is removed by requiring $\log (z-\zeta)$ to be real when $z-\zeta>0$.

The general fractional calculus operators $I_{0, z}^{\lambda, \mu, \eta}$ and $J_{0, z}^{\lambda, \mu, \eta}$ include (as their special cases) the Riemann-Liouville and the Erdélyi-Kober operators of fractional calculus studied by Saigo [8] as well as Srivastava and Saigo [11] and (more recently) by Atshan [1]. In fact, Atshan [1] made use of the general fractional calculus operator $I_{0, z}^{\lambda, \mu, \eta}$ in his investigation of a class of analytic and univalent functions which he defined by means of the celebrated Hovlov convolution operator involving the Gauss hypergeometric function ${ }_{2} F_{1}(a, b ; c ; z)$ in $(7)$. For a systematic investigation of such much more general convolution 
operators as the Dziok-Srivastava operator and the Srivastava-Wright operator, the reader is referred to an interesting recent work by Kiryakova [3].

It is easy to observe that (see [7])

$$
J_{0, z}^{\lambda, \mu, \eta} f(z)=\frac{d}{d z}\left(I_{0, z}^{1-\lambda, \mu-1, \eta-1} f(z)\right) \quad(0 \leqq \lambda<1 ; \mu, \eta \in \mathbb{R}) .
$$

\section{A Set of Preliminaries}

In order to prove our main results for functions belonging to the class $\mathcal{T}_{n}(\lambda, \mu, \eta)$, we shall need the following lemma.

Lemma 1. If $0 \leqq \lambda<1, \mu, \eta \in \mathbb{R}$ and $\kappa>\max \{0, \mu-\eta\}-1$, then

$$
J_{0, z}^{\lambda, \mu, \eta} z^{\kappa}=\frac{\Gamma(\kappa+1) \Gamma(\kappa-\mu+\eta+1)}{\Gamma(\kappa-\mu+1) \Gamma(\kappa-\lambda+\eta+1)} z^{\kappa-\mu} .
$$

Proof. The assertion (14) of Lemma 1 is due essentially to Srivastava et al. [12, p. 415, Lemma 3]. Their demonstration of the assertion (14) makes use of the following known results (see [9, p. 287, Eq. 9.4 (44)]):

$$
\begin{aligned}
{ }_{2} F_{1}(\alpha, \beta ; \gamma ; z)= & \frac{\Gamma(\gamma)}{\Gamma(\lambda) \Gamma(\gamma-\lambda)} \int_{0}^{1} t^{\lambda-1}(1-t)^{\gamma-\lambda-1}{ }_{2} F_{1}(\alpha, \beta ; \lambda ; z t) d t \\
& (\Re(\gamma)>\Re(\lambda)>0 ; z \neq 1 ;|\arg (1-z)|<\pi)
\end{aligned}
$$

and (see [9, p. 19, Eq. $1.2(20)])$

$$
{ }_{2} F_{1}(\alpha, \beta ; \gamma ; 1)=\frac{\Gamma(\gamma) \Gamma(\gamma-\alpha-\beta)}{\Gamma(\gamma-\alpha) \Gamma(\gamma-\beta)} \quad\left(\Re(\gamma-\alpha-\beta)>0 ; \gamma \in \mathbb{C} \backslash \mathbb{Z}_{0}^{-}\right),
$$

where $\mathbb{Z}_{0}^{-}$denotes the set of non-positive integers (see also the related works [6] and [7]).

Lemma 2. If $0 \leqq \lambda<1, \mu, \eta \in \mathbb{R}$ and $\kappa>\max \{0, \mu-\eta\}-1$, then

$$
J_{0, z}^{\lambda+1, \mu+1, \eta+1} z^{\kappa}=\frac{(\kappa-\mu) \Gamma(\kappa+1) \Gamma(\kappa-\mu+\eta+1)}{(\kappa-\mu+1) \Gamma(\kappa-\lambda+\eta+1)} z^{\kappa-\mu-1} .
$$


Proof. The assertion (17) of Lemma 2 is a simple consequence of Lemma 1.

\section{A Characterization Property}

We investigate the following characterization property for a function $f(z)$ belonging to the class $\mathcal{T}_{n}(\lambda, \mu, \eta)$ by means of coefficient bounds.

Theorem 1. A function $f(z)$ defined by (1) is in the class $\mathcal{T}_{n}(\lambda, \mu, \eta)$ if and only if

$$
\begin{aligned}
\sum_{k=n+1}^{\infty} & \frac{[k+\alpha\{\lambda(k-\mu-1)\}-1] \Gamma(k+1) \Gamma(k-\mu+\eta+1)}{\Gamma(k-\mu+1) \Gamma(k-\lambda+\eta+1)} a_{k} \\
\leqq & \frac{\alpha(1-\lambda \mu) \Gamma(\eta-\mu+2)}{\Gamma(2-\mu) \Gamma(\eta-\lambda+2)} \\
& (0 \leqq \lambda<1 ; \mu, \eta \in \mathbb{R} ; \varepsilon>\max \{0, \mu-\eta\}-1) .
\end{aligned}
$$

The result is sharp.

Proof. First of all, it can be seen from Lemma 1 that

$$
\begin{aligned}
& J_{0, z}^{\lambda, \mu, \eta} z^{k}=\frac{\Gamma(k+1) \Gamma(k-\mu+\eta+1)}{\Gamma(k-\mu+1) \Gamma(k-\lambda+\eta+1)} z^{k-\mu} \\
& (0 \leqq \lambda<1 ; \mu, \eta \in \mathbb{R} ; k>\max \{0, \mu-\eta\}-1) .
\end{aligned}
$$

We now suppose that the function $f(z) \in \mathcal{T}_{n}(\lambda, \mu, \eta)$ is given by $(1)$ and that the inequality (3) holds true. We then find from (14) that

$$
\begin{aligned}
& \left|z^{\mu} J_{0, z}^{\lambda+1, \mu+1, \eta+1} f(z)-(1-\mu) J_{0, z}^{\lambda, \mu, \eta} f(z)\right| \\
& -\alpha\left|z^{\mu} \lambda J_{0, z}^{\lambda+1, \mu+1, \eta+1} f(z)+(1-\lambda) J_{0, z}^{\lambda, \mu, \eta} f(z)\right| \\
& =\left|(1-k) \sum_{k=n+1}^{\infty} \Theta(\lambda, \mu, \eta) a_{k} z^{k-1}\right| \\
& -\alpha\left|\frac{(1-\lambda \mu) \Gamma(\eta-\mu+2)}{\Gamma(2-\mu) \Gamma(\eta-\mu+2)}-\sum_{k=n+1}^{\infty}[k+\alpha\{\lambda(k-\mu-1)\}-1] \Theta(\lambda, \mu, \eta) a_{k} z^{k-1}\right|
\end{aligned}
$$




$$
\begin{aligned}
& \sum_{k=n+1}^{\infty}(k-1)+\alpha[\lambda(k-\mu-1)+1] \Theta(\lambda, \mu, \eta) a_{k}-\frac{\alpha(1-\lambda \mu) \Gamma(\eta-\mu+2)}{\Gamma(2-\mu) \Gamma(\eta-\lambda+2)} \\
& \quad \leqq 0
\end{aligned}
$$

where

$$
\Theta(\lambda, \mu, \eta):=\frac{\Gamma(k+1) \Gamma(k-\mu+\eta+1)}{\Gamma(k-\mu+1) \Gamma(k-\lambda+\eta+1)}
$$

Hence, by the Maximum Modulus Theorem, we conclude that $f(z) \in \mathcal{T}_{n}(\lambda, \mu, \eta)$.

To prove the converse, we assume that the function $f(z)$ is defined by (1) and is in the class $\mathcal{T}_{n}(\lambda, \mu, \eta)$. Then the condition (3.1) readily yields

$$
\begin{aligned}
& \left|\frac{z J_{0, z}^{\lambda+1, \mu+1, \eta+1} f(z)-(1-\mu) J_{0, z}^{\lambda, \mu, \eta} f(z)}{\lambda z J_{0, z}^{\lambda+1, \mu+1, \eta+1} f(z)+(1-\lambda) J_{0, z}^{\lambda, \mu, \eta} f(z)}\right| \\
& =\left|-\frac{\sum_{k=n+1}^{\infty}(k-1) \Theta(\lambda, \mu, \eta) a_{k} z^{k-1}}{\frac{(1-\lambda \mu) \Gamma(\eta-\mu+2)}{\Gamma(2-\mu) \Gamma(\eta-\lambda+2)}-\sum_{k=n+1}^{\infty}[\lambda(k-\mu-1)+1] \Theta(\lambda, \mu, \eta) a_{k} z^{k-1}}\right| \\
& =\left|-\frac{\sum_{k=n+1}^{\infty} \frac{(k-1) \Theta(\lambda, \mu, \eta) \Gamma(2-\mu) \Gamma(\eta-\lambda+2)}{\Gamma(\eta-\mu+2)} a_{k} z^{k-1}}{(1-\lambda \mu)-\sum_{k=n+1}^{\infty} \frac{[\lambda(k-\mu-1)+1] \Theta(\lambda, \mu, \eta) \Gamma(2-\mu) \Gamma(\eta-\mu+2)}{\Gamma(\eta-\mu+2)} a_{k} z^{k-1}}\right| \\
& <\alpha \text {. }
\end{aligned}
$$

Since $|\Re(z)| \leqq|z| \quad(z \in \mathbb{C})$, if we choose $z$ to be real and let $z \rightarrow 1-$, we get

$$
\begin{aligned}
& \sum_{k=n+1}^{\infty} \frac{(k-1) \Theta(\lambda, \mu, \eta) \Gamma(2-\mu) \Gamma(\eta-\lambda+2)}{\Gamma(\eta-\mu+2)} \\
& \quad \leqq \alpha\left((1-\lambda \mu)-\sum_{k=n+1}^{\infty} \frac{[\lambda(k-\mu-1)+1] \Theta(\lambda, \mu, \eta) \Gamma(2-\mu) \Gamma(\eta-\mu+2)}{\Gamma(\eta-\mu+2)}\right),
\end{aligned}
$$

so that

$$
\sum_{k=n+1}^{\infty}[k+\alpha\{\lambda(k-\mu-1)\}-1] \Theta(\lambda, \mu, \eta) \leqq \frac{\alpha(1-\lambda \mu) \Gamma(\eta-\mu+2)}{\Gamma(2-\mu) \Gamma(\eta-\lambda+2)}
$$

which evidently complete the proof of Theorem 1 . 
Corollary. If function $f(z)$ defined by $(1)$ is in the class $\mathcal{T}_{n}(\lambda, \mu, \eta)$, then

$$
\begin{aligned}
& a_{k} \leqq \\
& \frac{\alpha(1-\lambda \mu) \Gamma(\eta-\mu+2) \Gamma(k-\mu+1) \Gamma(k-\lambda+\eta+1)}{[k+\alpha\{\lambda(k-\mu-1)+1\}-1] \Gamma(2-\mu) \Gamma(\eta-\lambda+2) \Gamma(k+1) \Gamma(k-\mu+\eta+1)} \\
& (0 \leqq \lambda<1 ; \mu, \eta \in \mathbb{R} ; k>\max \{0, \mu-\eta\}-1) .
\end{aligned}
$$

Remark 1. By suitably specializing or modifying the parameters involved in Theorem 1, we can derive the characterization properties corresponding to the fractional calculus operators $D_{z}^{\lambda} f(z)$ and $I_{0, z}^{\lambda, \mu, \eta} f(z)$.

\section{A Distortion Theorem}

In this section, we prove the following distortion theorem involving the fractional calculus operator $J_{0, z}^{\lambda, \mu, \eta}$ defined by (12).

Theorem 2. If $f(z) \in \mathcal{T}_{n}(\lambda, \mu, \eta)$, then

$$
\begin{gathered}
\left|J_{0, z}^{\lambda, \mu, \eta} f(z)\right| \leqq \frac{\Gamma(\eta-\mu+2)|z|^{1-\mu}}{\Gamma(2-\mu) \Gamma(\eta-\lambda+2)}\left(1+\Phi(n+1)|z|^{n} \sum_{k=n+1}^{\infty} \Gamma(k+1) a_{k}\right) \\
(z \in \mathbb{U} ; 0 \leqq \lambda<1 ; \mu, \eta \in \mathbb{R})
\end{gathered}
$$

and

$$
\begin{gathered}
\left|J_{0, z}^{\lambda, \mu, \eta} f(z)\right| \geqq \frac{\Gamma(\eta-\mu+2)|z|^{1-\mu}}{\Gamma(2-\mu) \Gamma(\eta-\lambda+2)}\left(1-\Phi(n+1)|z|^{n} \sum_{k=n+1}^{\infty} \Gamma(k+1) a_{k}\right), \\
(z \in \mathbb{U} ; 0 \leqq \lambda<1 ; \mu, \eta \in \mathbb{R}),
\end{gathered}
$$

where

$$
\Phi(n):=\frac{\Gamma(n-\mu+\eta+1)}{\Gamma(n-\mu+1) \Gamma(n-\lambda+\eta+1)} .
$$


Proof. Since $f(z) \in \mathcal{T}_{n}(\lambda, \mu, \eta)$, by applying the assertion (18), we obtain

$$
\begin{aligned}
& \frac{n+\{\lambda(\eta-\mu)\} \Gamma(\eta-\mu+n+2)}{\Gamma(n-\mu+2) \Gamma(\eta-\lambda+n+2)} \sum_{k=n+1}^{\infty} \Gamma(k+1) a_{k} \\
& \quad \leqq \sum_{k=n+1}^{\infty} \frac{[k+\alpha\{\lambda(k-\mu-1)+1\}-1] \Gamma(k-\mu+\eta+1)}{\Gamma(k+-\mu+1) \Gamma(k-\lambda+\eta+1)} a_{k} \\
& \leqq \frac{\alpha(1-\lambda \mu) \Gamma(\eta-\mu+2)}{\Gamma(2-\mu) \Gamma(\eta-\lambda+2)}
\end{aligned}
$$

which immediately yields

$$
\sum_{k=n+1}^{\infty} \Gamma(k+1) a_{k} \leqq \frac{\alpha(1-\lambda \mu) \Gamma(\eta-\mu+2) \Gamma(n-\mu+2) \Gamma(\eta-\lambda+n+2)}{\Gamma(2-\mu)[n+\{\lambda(\eta-\mu)\}] \Gamma(\eta-\mu+n+2) \Gamma(\eta-\lambda+2)} .
$$

Now, making use of Definition 5, we get

$$
J_{0, z}^{\lambda, \mu, \eta} f(z)=\frac{\Gamma(\eta-\mu+2)}{\Gamma(2-\mu) \Gamma(\eta-\lambda+2)} z^{1-\mu}\left(1-\sum_{k=n+1}^{\infty} \Phi(k) \Gamma(k+1) a_{k} z^{k-1}\right),
$$

where

$\Phi(k):=\frac{\Gamma(k-\mu+\eta+1)}{\Gamma(k-\mu+1) \Gamma(k-\lambda+\eta+1)} \quad(k=n+1, n+2, n+3, \cdots ; n \in \mathbb{N})$.

Since $\Phi(k)$ is decreasing in $k$, we have

$$
0<\Phi(n) \leqq \Phi(n+1)=\frac{\Gamma(n-\mu+\eta+2)}{\Gamma(n-\mu+2) \Gamma(n-\lambda+\eta+2)} .
$$

From (27), (28) and (29), it is easily seen that

$$
\begin{aligned}
& \left|J_{0, z}^{\lambda, \mu, \eta} f(z)\right| \leqq \frac{\Gamma(\eta-\mu+2)|z|^{1-\mu}}{\Gamma(2-\mu) \Gamma(\eta-\lambda+2)}\left(1+\Phi(n+1)|z|^{n} \sum_{k=n+1}^{\infty} \Gamma(k+1) a_{k}\right) \\
& \leqq \frac{\Gamma(\eta-\mu+2)|z|^{1-\mu}}{\Gamma(2-\mu) \Gamma(\eta-\lambda+2)}\left(1+\frac{\alpha(1-\lambda \mu) \Gamma(\eta-\mu+2)}{\Gamma(2-\mu) \Gamma(\eta-\lambda+2)[n+\alpha(\lambda(n-\mu))]}|z|^{n}\right)
\end{aligned}
$$

and

$$
\left|J_{0, z}^{\lambda, \mu, \eta} f(z)\right| \geqq \frac{\Gamma(\eta-\mu+2)|z|^{1-\mu}}{\Gamma(2-\mu) \Gamma(\eta-\lambda+2)}\left(1-\Phi(n+1)|z|^{n} \sum_{k=n+1}^{\infty} \Gamma(k+1) a_{k}\right)
$$




$$
\geqq \frac{\Gamma(\eta-\mu+2)|z|^{1-\mu}}{\Gamma(2-\mu) \Gamma(\eta-\lambda+2)}\left(1-\frac{\alpha(1-\lambda \mu) \Gamma(\eta-\mu+2)}{\Gamma(2-\mu) \Gamma(\eta-\lambda+2)[n+\alpha(\lambda(n-\mu))]}|z|^{n}\right) .
$$

These last inequalities evidently complete the proof of Theorem 2 .

Remark 2. By suitably specializing or modifying the parameters involved in Theorem 2, we can derive distortion theorems for the fractional calculus operators $D_{z}^{\lambda} f(z)$ and $I_{0, z}^{\lambda, \mu, \eta} f(z)$.

\section{Radii of Close-To-Convexity, Starlikeness and Convexity}

A function $f(z) \in \mathcal{T}_{n}$ is said to be close-to-convex of order $\rho$ in $\mathbb{U}$ if

$$
\Re\left(f^{\prime}(z)\right)>\rho \quad(0 \leqq \rho<1 ; \forall z \in \mathbb{U}) .
$$

If $f(z) \in \mathcal{T}_{n}$ satisfies the following inequality:

$$
\Re\left(\frac{z f^{\prime}(z)}{f(z)}\right)>\rho \quad(0 \leqq \rho<1 ; \forall z \in \mathbb{U}),
$$

then the function $f(z)$ is said to be starlike of order $\rho$ in $\mathbb{U}$. On the other hand, if $f(z) \in \mathcal{T}_{n}$ satisfies the following inequality:

$$
\Re\left(1+\frac{z f^{\prime \prime}(z)}{f^{\prime}(z)}\right)>\rho \quad(0 \leqq \rho<1 ; \forall z \in \mathbb{U}),
$$

then the function $f(z)$ is said to be convex of order $\rho$ in $\mathbb{U}$ (see, for details, [2]).

We now prove the following theorems.

Theorem 3. If a function $f(z) \in \mathcal{T}_{n}$, then $f(z)$ is close-to-convex of order $\rho(0 \leqq \rho<1)$ in $|z|<r_{1}$, where

$$
\begin{aligned}
& r_{1}=r_{1}(\lambda, \mu, \eta, \rho)=\inf _{k \geqq n+1} \\
& \left(\frac{(1-\rho)[k+\alpha\{\lambda(k-\mu-1)\}-1] \Theta(\lambda, \mu, \eta) \Gamma(2-\mu) \Gamma(\eta-\lambda+2)}{\alpha(1-\lambda \mu) \Gamma(\eta-\mu+2)}\right)^{\frac{1}{1-k}},
\end{aligned}
$$

where $\Theta(\lambda, \mu, \eta)$ is given by $(21)$. 
Proof. Let the function $f(z) \in \mathcal{T}_{n}(\lambda, \mu, \eta)$ be given by (1). Then, by virtue of (30), the function $f(z)$ is close-to-convex of order $\rho$ in $\mathbb{U}$, provided that

$$
\begin{aligned}
\left|f^{\prime}(z)-1\right| & =\left|\sum_{k=n+1}^{\infty} k a_{k} z^{k-1}\right| \\
& \leqq \sum_{k=n+1}^{\infty} k a_{k}|z|^{k-1} \\
& \leqq 1-\rho \quad(k \geqq n+1 ; n \in \mathbb{N}) .
\end{aligned}
$$

Now, in view of (18), the assertion (34) holds true if

$$
\begin{gathered}
\frac{k|z|^{k-1}}{1-\rho} \leqq \frac{[k+\alpha\{\lambda(k-\mu-1)\}-1] \Theta(\lambda, \mu, \eta) \Gamma(2-\mu) \Gamma(\eta-\lambda+2)}{\alpha(1-\lambda \mu) \Gamma(\eta-\mu+2)} \\
(k \geqq n+1 ; n \in \mathbb{N}) .
\end{gathered}
$$

Finally, upon solving (35) for $|z|$, we readily obtain the assertion (33) of Theorem 3.

Theorem 4. If a function $f(z) \in \mathcal{T}_{n}$, then $f(z)$ is starlike of order $\rho(0 \leqq$ $\rho<1)$ in $|z|<r_{2}$, where

$$
\begin{aligned}
& r_{2}=r_{2}(\lambda, \mu, \eta, \rho)=\inf _{k \geqq n+1} \\
& \left(\frac{(1-\rho)[k+\alpha\{\lambda(k-\mu-1)\}-1] \Theta(\lambda, \mu, \eta) \Gamma(2-\mu) \Gamma(\eta-\lambda+2)}{\alpha(1-\lambda \mu) \Gamma(\eta-\mu+2)}\right)^{\frac{1}{1-k}},
\end{aligned}
$$

where $\Theta(\lambda, \mu, \eta)$ is given by (21).

Proof. Suppose that $f(z) \in \mathcal{T}_{n}(\lambda, \mu, \eta)$ is given by (1). Then, in light of (30), the function $f(z)$ is starlike of order $\rho$ in $\mathbb{U}$, provided that

$$
\begin{aligned}
\left|\frac{z f^{\prime}(z)}{f(z)}-1\right| & =\left|\frac{\sum_{k=n+1}^{\infty}(k-1) a_{k} z^{k-1}}{1-\sum_{k=n+1}^{\infty} a_{k} z^{k-1}}\right| \\
& \leqq \frac{\sum_{k=n+1}^{\infty}(k-1) a_{k}|z|^{k-1}}{1-\sum_{k=n+1}^{\infty} a_{k}|z|^{k-1}}
\end{aligned}
$$




$$
\leqq 1-\rho \quad(k \geqq n+1 ; n \in \mathbb{N})
$$

In view of (18), this last assertion (37) holds true if

$$
\begin{gathered}
\frac{(k-\rho)|z|^{k-1}}{1-\rho} \leqq \frac{[k+\alpha\{\lambda(k-\mu-1)\}-1] \Theta(\lambda, \mu, \eta) \Gamma(2-\mu) \Gamma(\eta-\lambda+2)}{\alpha(1-\lambda \mu) \Gamma(\eta-\mu+2)} \\
(k \geqq n+1 ; n \in \mathbb{N}),
\end{gathered}
$$

$\Theta(\lambda, \mu, \eta)$ is given by (21). Thus, upon solving (38) for $|z|$, we are led easily to the assertion (36) of Theorem 4.

Theorem 5. If a function $f(z) \in \mathcal{T}_{n}$, then $f(z)$ is convex of order $\rho(0 \leqq$ $\rho<1)$ in $|z|<r_{3}$, where

$$
\begin{aligned}
& r_{3}=r_{3}(\lambda, \mu, \eta, \rho)=\inf _{k \geqq n+1} \\
& \left(\frac{(1-\rho)[k+\alpha\{\lambda(k-\mu-1)\}-1] \Theta(\lambda, \mu, \eta) \Gamma(2-\mu) \Gamma(\eta-\lambda+2)}{\alpha(1-\lambda \mu) \Gamma(\eta-\mu+2)}\right)^{\frac{1}{1-k}},
\end{aligned}
$$

where $\Theta(\lambda, \mu, \eta)$ is given by $(21)$.

Proof. Let $f(z) \in \mathcal{T}_{n}(\lambda, \mu, \eta)$ be given by (1). Then, in view of (30), the function $f(z)$ is convex of order $\rho$ in $\mathbb{U}$, provided that

$$
\begin{aligned}
\left|\frac{z f^{\prime \prime}(z)}{f^{\prime}(z)}\right| & =\left|\frac{\sum_{k=n+1}^{\infty} k(k-1) a_{k} z^{k-1}}{1-\sum_{k=n+1}^{\infty} k a_{k} z^{k-1}}\right| \\
& \leqq \frac{\sum_{k=n+1}^{\infty} k(k-1) a_{k}|z|^{k-1}}{1-\sum_{k=n+1}^{\infty} k a_{k}|z|^{k-1}} \\
& \leqq 1-\rho \quad(k \geqq n+1 ; n \in \mathbb{N}) .
\end{aligned}
$$

By appealing to (18), the last assertion (40) holds true if

$$
\frac{k(k-\rho)|z|^{k-1}}{1-\rho} \leqq \frac{[k+\alpha\{\lambda(k-\mu-1)\}-1] \Theta(\lambda, \mu, \eta) \Gamma(2-\mu) \Gamma(\eta-\lambda+2)}{\alpha(1-\lambda \mu) \Gamma(\eta-\mu+2)}
$$




$$
(k \geqq n+1 ; n \in \mathbb{N}),
$$

where $\Theta(\lambda, \mu, \eta)$ is given by (21).

Finally, upon solving the equation (42) for $|z|$, we get the assertion (39) of Theorem 5 .

Remark 3. By suitably specializing or modifying the parameters involved in Theorem 3, Theorem 4 and Theorem 5, it is fairly straightforward to derive the radii of close-to-convexity, starlikeness and convexity corresponding to the fractional calculus operators $D_{z}^{\lambda} f(z)$ and $I_{0, z}^{\lambda, \mu, \eta} f(z)$.

\section{References}

[1] W. G. Atshan, Applications of fractional calculus operators for a new class of univalent functions with negative coefficients defined by Hohlov operator, Math. Slovaca 60 (2010), 75-82.

[2] P. L. Duren, Univalent Functions, Grundlehren der Mathematischen Wissenschaften, Band 259, Springer-Verlag, New York, Berlin, Heidelberg and Tokyo, 1983.

[3] V. Kiryakova, Criteria for univalence of the Dziok-Srivastava and the Srivastava-Wright operators in the class $\mathcal{A}$, Appl. Math. Comput. 218 (2011), 883-892.

[4] S. Owa, On the distortion theorem. I, Kyungpook Math. J. 18 (1978), $53-59$.

[5] S. Owa and H. M. Srivastava, Univalent and starlike generalized hypergeometric functions, Canad. J. Math. 39 (1987), 1057-1077.

[6] R. K. Raina and H. M. Srivastava, A certain subclass of analytic functions associated with operators of fractional calculus, Comput. Math. Appl. 32 (7) (1996), 13-19.

[7] R. K. Raina and H. M. Srivastava, Some subclasses of analytic functions associated with fractional calculus operators, Comput. Math. Appl. 37 (1999), $73-84$. 
[8] M. Saigo, A remark on integral operators involving the Gauss hypergeometric functions, Math. Rep. College General Ed. Kyushu Univ. 11 (1978), $135-143$.

[9] H. M. Srivastava and P. W. Karlsson, Multiple Gaussian Hypergeometric Series, Halsted Press (Ellis Horwood Limited, Chichester), John Wiley and Sons, New York, Chichester, Brisbane and Toronto, 1985.

[10] H. M. Srivastava and S. Owa, An application of the fractional derivative, Math. Japon. 29 (1984), 383-389.

[11] H. M. Srivastava and M. Saigo, Multiplication of fractional calculus operators and boundary value problems involving the Euler-Darboux equation, J. Math. Anul. Appl. 121 (1987), 325-369.

[12] H. M. Srivastava, M. Saigo and S. Owa, A class of distortion theorems involving certain operators of fractional calculus, J. Math. Anal. Appl. 131 (1988), 412-420. 